\title{
Soluciones de humanización en salud en la práctica diaria
}

\section{Humanization Solutions health in daily practice}

Dra. Claudia Ariza Olarte

\section{Resumen}

El presente artículo encierra una reflexión con relación a la importancia, que desde el punto de vista del autor, tiene para todos los profesionales de la salud, proporcionar una atención y o cuidado, que se caracterice por ser humano. El objetivo fue plantear los procesos que contribuyen a la humanización de los servicios de salud, la ciencia y la tecnología y los atributos que deben caracterizar la atención que se brinda en ellos para que ésta sea humana, teniendo en cuenta los diferentes agentes que participan en el desarrollo de asistencia en salud: profesionales, pacientes y familias.

Para darle alcance a este objetivo se realizó una revisión de la literatura del sector de la salud relacionada con el tema, incluyendo disciplinas como Medicina y Enfermería dentro de la que se tuvo en cuenta la Ley 911 de 2004, por la cual se dictan disposiciones en materia de responsabilidad deontológica para el ejercicio de la Enfermería en Colombia, se establece el régimen disciplinario correspondiente y se dictan otras disposiciones.

Las acciones de profesionales e instituciones asistenciales y formadoras, deberían tender a favorecer que la atención humana se caracterice por estar matizada de valores éticos y morales dentro de los cuales el respeto ocupa un lugar privilegiado. Esto implica además dar una dirección al desarrollo tecnológico, una dirección que habrá de conducirlo de vuelta a las necesidades reales del hombre.

Enfermera Cardióloga. Doctora en Enfermería. Profesora Asociada.

Facultad de Enfermería. Facultad de Medicina. Pontificia Universidad Javeriana. Bogotá. Colombia

Fecha de Recibido: 3 De Septiembre 2011

Fecha de EnVIAdo: 15 DE OCTUbRe 2011

Fecha De ACeptado: 4 DE Diciembre 2011 


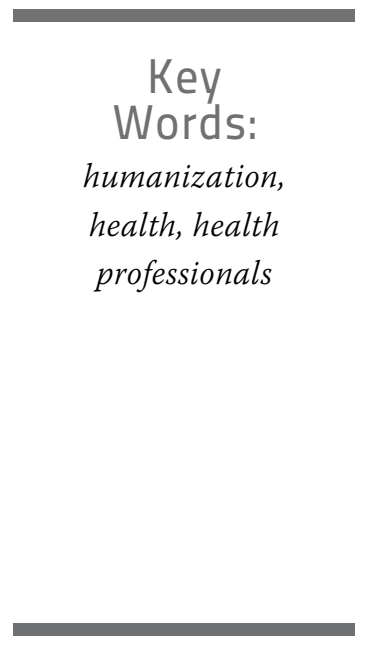

AbStrac

This article contains a reflection with respect to the importance that, from for the standpoint of the author, has for all health professionals, providing attention and or care, which must be characterized for being humane. The objective was to assess the processes that contribute to the humanization of health care, science and technology and the attributes that should characterize the care provided to them for it be humane, taking into account the different actors involved in the development of health care: professionals, patients and families.

To give scope to this objective a review of the literature of the health sector related to the topic was made, including disciplines such as medicine and nursing, in which was taken into account the 911 Law of 2004, by enacting provisions on ethical liability for the exercise of nursing in Colombia, determining the appropriate disciplinary and other provisions.

The actions of health care professionals and institutions, and trainers, should tend to favor humane attention to be characterized for being laden with ethical and moral values in which respect occupies a privileged place. This also implies giving a direction to technological development, one that will lead it back to the real needs of man.

\section{INTRODUCCIÓN}

Una de las mayores preocupaciones que afrontan los profesionales, gestores y usuarios de los sistemas de salud, es la humanización de los servicios de salud, tal y como lo plantean las diferentes normativas y documentos. ${ }^{1}$

Cuando se habla de humanizar la atención en salud, se hace referencia, en principio, a todas aquellas acciones que hacen más humano al hombre; por ello, es necesario conocer el concepto de humanización que tienen los diferentes agentes que participan en el proceso de asistencia en salud: profesionales, pacientes y familias.Del concepto que cada profesional de la salud tenga acerca de qué y quién es el hombre, depende la manera como se conduzca al brindar la atención.

En cuanto a los procesos de humanización hay que partir de quiénes son los trabajadores del área de la salud: médicos, enfermeras, terapistas, nutricionistas, bacteriólogos, entre otros. A ellos es importante recordarles que están llamados a poner todo su conocimiento al servicio de los demás, sin diferencia del nivel en que se encuentren,poniendo en primer lugar la sensibilidad y la ética en el acto del cuidado resaltando la dignidad humana y ofreciendo una óptima atención, comunicación e información a los usuarios, los pacientes, sus familias y otros profesionales involucrados en el sistema de salud.

El respeto a la vida, a la dignidad de los seres humanos y a sus derechos, sin distinción de edad, credo, sexo, raza, nacionalidad, lengua, cultura, condición socioeconómica e ideología política, son principios $y$ valores fundamentales que orientan el ejercicio de la enfermería y que pueden ser aplicados al ejercicio de todos los profesionales de la salud, para hacer que éste sea humano. Se da a partir de la comunicación y relación interpersonal humanizada entre el profesional de la salud y el ser humano, sujeto de cuidado, la familia o grupo social, en las distintas etapas de la vida, situación de salud y del entorno. ${ }^{2}$

La atención implica un juicio de valor y un proceso dinámico $y$ participativo para identificar y dar prioridad a las necesidades y decidir la intervención médica, el plan de cuidado de enfermería y o de otros profesionales de la salud, con el propósito de promover la vida, prevenir la enfermedad, intervenir en el tratamiento, en la rehabilitación y dar cuidado paliativo con el fin de desarrollar, en lo posible, las potencialidades individuales y colectivas. ${ }^{2}$ 


\section{DesarRollo}

Para hablar de humanización de los servicios de salud e incorporar el conjunto de valores implicados en esta, es importante en primer lugar, hacer un llamado a las instituciones del sector salud, como responsables de proveer a su personal profesional y técnico de las herramientas e insumos para que puedan cumplir apropiadamente sus funciones,no solamente considerar las deficiencias en la formación y en las relaciones interpersonales, sino también las limitaciones y carencias de infraestructura y equipos que influyen en una atención de calidad y que se unen a la falta de preparación y actualización, mediando en el logro de los resultados, objetivos y metas.Entiéndase por condicionespara el ejercicio profesional, los requisitos básicos indispensables de personal, infraestructura física, dotación, procedimientos técnico-administrativos, registros para el sistema de información, transporte, comunicaciones, auditoría de servicios y medidas de seguridad, que le permitan al profesional actuar con autonomía profesional, calidad e independencia y sin los cuales no podrá dar garantía del acto de atención.

Entonces, los profesionales de la salud que brinden una atención humana, deberán garantizar la calidad de la misma a quienes reciben sus servicios. Tal garantía no debe entenderse en relación con los resultados de la intervención profesional, dado que el ejercicio implica una obligación de medios, mas no de resultados. La valoración ética de la atención deberá tener en cuenta las circunstancias de tiempo, modo y lugar que rodearon los hechos y las precauciones que frente al mismo hubiera aplicado un profesional de la salud prudente y diligente. ${ }^{2}$

Las instituciones deben buscar de manera permanente los mecanismos para motivarlos e incentivarlos para que sientan gusto y placer de ejercer su profesión, así como suprimir las barreras organizativas y administrativas innecesarias, para alcanzar una resolución eficiente y de excelencia. Asimismo sería importante considerar las condiciones laborales que actualmente dichas instituciones ofrecen: contratos por tiempo limitado lo cual produce incertidumbre laboral, salarios insuficientes que conllevan a la necesidad de tener que contar con varios empleos a la vez, recargo de responsabilidades administrativas que impiden en algunas ocasiones, poder dedicar el tiempo justo a cada una de las personas que solicitan la atención en salud.

En segundo término, a estas entidades están muy ligadas las instituciones universitarias formadoras, quienes además de transmitir los conocimientos científicos, están llamadas a inculcar los valores morales y éticos como eje transversal a través de todo el proceso formativo, con el propósito de lograr la excelencia y el mejor adiestramiento posible.

Este proceso de humanización de las instituciones es parte fundamental de la calidad del servicio que se presta. Para que la atención brindada por dichas instituciones de salud, sea de calidad el grupo de profesionales encargado de hacerlo, debe tener en cuenta su preparación, sus conocimientos, no solamente desde el punto de vista científico sino desde todas las dimensiones del hombre: costum- bres, cultura, creencias religiosas, etc., las cuales en un momento determinado caracterizarán la atención que éste requiera. Por esta razón el estudio y la investigación que deben caracterizar a todos los profesionales de la salud, proporcionan herramientas que conducen a un conocimiento científico, moral y humano óptimos para ofrecer una atención dirigida no solo a la esfera biológica de la persona, sino también a sus otras dimensiones, las cuales tratadas en conjunto conducen a su cuidado integral, incluir a su familia y o comunidad, lo cual implica contemplar la dignidad humana. ${ }^{3}$

La dignidad como algo propio de la persona humana debe cumplirse en relación a sí mismo y en relación a los demás. Kant planteaba que existe la obligación estricta de salvaguardar y hacer crecer la propia dignidad personal, si no es así se defrauda a sí mismo y a la humanidad entera. ${ }^{4,5}$

Y entonces se podría preguntar: Por qué hablar de dignidad humana? Precisamente porque, a todo lo que hasta ahora se ha dicho acerca de las responsabilidades de las instituciones prestadoras de servicios y de aquellas encargadas de la formación de los profesionales de la salud, se anteponeque unas y otras deben tener permanente conciencia de que el servicio que prestan y la formación que reciben, debe estar a disposición de la PERSONA.

Además, en doble sentido, el respeto debe darse en cuanto al ser propio y ajeno, al obrar, y a la libertad del otro. Respeto al ser propio y ajeno con todas sus dimensiones, incluidas las corpóreas, puesto que el cuerpo humano es también personal. Respeto al obrar correspon- 
diente a la condición de persona en uno mismo y en los demás, es cierto que en virtud del ser personal que se detenta y del que no se puede prescindir, se tiene la obligación de desarrollar las capacidades hasta dar de sí mismo todo lo que se pueda. ${ }^{6}$

Entonces, el respeto, es una actitud que debe adoptarse tanto con las personas como con las cosas. Constituye un reclamo de la dignidad de la persona calificado como veneración o reverencia. Agustín de Hipona y Séneca fundamentaban la veneración debida al enfermo calificando a éste como "re sacar miser", es decir, algo sagrado en un estado miserable. La índole "sagrada" del paciente está clara, y constituye el trasunto de su nobleza personal. La condición dolorosa, no añade un incremento de excelencia respecto al individuo sano, pero parece exigir mayor veneración y miramiento en el trato con él, precisamente por su extrema vulnerabilidad. Debido a que la dignidad del paciente se encuentra de manera temporal amenazada, ésta reclama un suplemento de consideración y respeto. Para conocer y reconocer en alguien a una persona es imprescindible la actitud, básica y previa, de estar dispuesto a hacerlo, con todas las consecuencias prácticas que eso lleve consigo. ${ }^{7}$

Cabría entonces enfatizar que el usuario, el paciente y o su familia, ante todo son personas, es decir, que no solamente tienen una dimensión somática, psicológica y sociocultural, poseen también una parte espiritual que les da unidad y los integra al mundo de los valores y principios fundamentales de la vida; son seres con dignidad que merecen respeto. Por ejemplo,decimos "el de la cama 8, el infarto complicado, la diabetes descompensada", en lugar de hablar de la persona que presenta estas alteraciones.

Para muchos la salud es hoy la principal preocupación de hombres y mujeres. La aplicación de nuevas tecnologías en las diferentes áreas del mundo de la salud, han contribuido a mejorar las condiciones de usuarios y pacientes. Sin embargo "ninguna máquina puede suprimir el efecto curador y o terapéutico de una mirada, de la palabra adecuada o del silencio oportuno. La presencia humana es insustituible en los procesos de enfermedad, dolor o muerte" (Centro de Humanización de la Salud, Madrid). ${ }^{8}$

El uso inapropiado de la tecnología puede llevar a creer que solo con ello es suficiente para causar satisfacción duradera en la persona a quien se atiende. Los avances en la tecnología han disminuido la necesidad de interrogatorios clínicos detallados o de exámenes clínicos exhaustivos, lo cual ha implicado la pérdida de importancia que se le daba al contacto del profesional de la salud con el paciente, deshumanizando y mecanizando la relación. Es importante recalcar el cuidado que deben tener estos profesionales en cuanto a mantener la calidez y la sensibilidad al dolor. La tecnología debe servir para mejorar la comunicación, para informar, para investigar, para divulgar, para capacitar, para lograr mejores diagnósticos y tratamientos, sin abandonar la relación profesional-paciente, como una relación persona a persona. ${ }^{8}$

Paterson y Zderad describen las características especiales de relacionarse derivadas de Buber para quien la relación YO-TU está marcada por un diálogo intuitivo e intersubjetivo verificado cuando un ser humano se involucra con otro. En esa relación sujeto-sujeto, cada uno reconoce las singularidades del otro, y ofrece al otro una presencia auténtica. Para estos autores, la autenticidad comprende además respuestas auditivas, olfativas, orales, visuales, y táctiles, cada una confiriendo un significado singular a la conciencia del otro. ${ }^{9}$ La relación YO-ESO de Buber comprende la relación sujeto-objeto; aunque guarde semejanza en la forma como las personas se integran con los objetos, sin embargo un objeto está abierto a la investigación, mientras que el ser humano como objeto puede darse a conocer $u$ obstaculizar ese conocimiento. Y la relación NOS es el fenómeno de la comunicación. Es el momento en que las personas se unen y luchan por un objetivo común; en este sentido Buber refiere que cada hombre puede ser comparado a la comunicación de sus TUS internalizados. ${ }^{9,10}$

Para Paterson en lo clínico se derivaba el cómo del trabajo hacia la comodidad. Es en lo clínico que la experimentación ocurre, siendo conscientes, después recordando, ponderando, clasificando y aprendiendo. Relacionando el YO-TU con el mundo clínico del otro, el clínico va, se entrega, y recibe de vuelta al otro y a sí mismo en la esfera del "entre". Conoce al otro y la mayoría de sí mismo en esta relación. Está confirmado y confirma al otro por medio de la presencia del otro. Así llama al otro a conocerlo a través de la relación clínica. En aceptar al otro como es, el clínico imagina y responde a la realidad de su potencial para 
convertirse, convertirse de acuerdo a su única capacidad de humanidad. ${ }^{11}$ Relacionando el YO-ESO con su mundo clínico, el clínico trasciende de sí mismo, se objetiviza y estudia el yo-tú que conoce. Clasifica y lo estudia. Le pregunta. Lo compara y lo contrasta con las otras experiencias clínicas. Discute sus muchos aspectos en diálogo con su interior y posiblemente variedad de exteriores. Reordena sus partes. Forma, crea, planea desde y para su existencia clínica. Así argumenta un mundo de conocimiento para encontrar respuestas". ${ }^{12}$

En últimas, la ciencia y la tecnología han facilitado el monitoreo fisiológico, el diagnóstico y el tratamiento del paciente obligando a estar alertas sobre alarmas, equipos, procedimientos invasivos, terapéuticas, etc. Pero también han alejado del aspecto emocional, afectivo y social de la persona enferma.

Cada vez se nos hacen exigencias mayores en el desarrollo de habilidades técnicas y no solo se permite sino que se contribuye a ello centrando el interés en la capacitación técnico-científica. Pero pocas veces se recuerda la importancia del contacto humano y la comunicación, lo cual limita que el desempeño en el trabajo sea íntegro, y al mismo tiempo lo basa en la alta tecnología olvidando el humanismo.

Por otra parte es importante mencionar que el mundo moderno ha sido modelado por la metafísica. La metafísica ha modelado la educación y esta, a su vez ha dado lugar a la ciencia y a la tecnología. Esto se puede resumir en que el mundo moderno ha sido modelado por la ciencia y la tecnología. Se tam- balea de crisis en crisis, por todos lados hay profecías de desastres y signos visibles de destrucción. ${ }^{13}$

Todo este panorama parece enfermo, podría ser conveniente observar por un momento la tecnología misma. Si se considera que ella se está convirtiendo cada vez más en algo inhumano, podría muy bien considerarse si existe la posibilidad de tener algo mejor: una tecnología y una ciencia con rostro humano. El problema no lo constituye la tecnología misma sino el uso que de ella hacemos los seres humanos. ${ }^{12}$

La principal tarea de la tecnología debe ser la de aliviar el peso del trabajo que el hombre tiene que llevar adelante para poder subsistir y desarrollar sus facultades potenciales.La tecnología moderna ha privado al hombre de la clase de trabajo que disfruta más, trabajo creativo, útil, hecho con sus manos y su cuerpo, y le ha dado abundante trabajo de un tipo fragmentado, la mayor parte del cual no le produce satisfacción. ${ }^{13}$

Aplicado esto a la situación de las instituciones de salud, se podría decir que se posee una vasta acumulación de nuevos conocimientos, técnicas científicas espléndidas para incrementarlas y una inmensa experiencia en su aplicación, Pero el uso que se le ha dado es apenas uno de los posibles usos y se puede ver que es un uso a menudo ignorante y destructivo. La experiencia por los diferentes campos de práctica, ha permitido observar con gran dolor, que muchos profesionales de la salud, han olvidado la esencia de su desempeño profesional que no es más que cuidar a la persona enferma. Al mismo tiempo esto les genera una gran inseguridad, la cual no puede dejarse identificar, razón per- fecta para dejarse arrastrar por los avances tecnológicos que invaden los servicios, ocultándose detrás de ellos y convirtiéndose en grandes técnicos. Entonces se debería preguntar ¿y el paciente dónde está? ¿Dónde está la razón de ser de la atención en salud?. ${ }^{13}$

No hay duda de que es posible dar una dirección al desarrollo tecnológico, una dirección que habrá de conducirlo de vuelta a las necesidades reales del hombre, lo que también significa volver al tamaño correcto del hombre. El hombre es pequeño y por tanto lo pequeño es hermoso. Hay que cambiar la tecnología de modo que le sirva al hombre y no que lo destruya. ${ }^{12}$

El profesional de la salud que todavía quiera desempeñarse como un técnico, se verá obligado a confesar que no ve en su paciente otra cosa que una máquina y no al ser humano que hay detrás de la enfermedad. El ser humano no es una cosa entre otras cosas, las cosas se determinan unas a otras, pero el hombre en última instancia es su propio determinante.

Ante el panorama descrito ¿Cuáles deben ser los atributos de la atención que brindan los profesionales de la salud para que ésta sea humana?

Es posible que en el inicio de las ciencias de la salud, se hiciera mayor énfasis en la exigencia de buena conducta, en la rectitud de las actuaciones, en observar las reglas de la etiqueta y urbanidad, en las relaciones interpersonales con el paciente, la familia y con el médico. ${ }^{13}$

Hoy se reconoce que la atención integral a la persona, la familia y grupos de la comunidad, es el objetivo primordial de los profesionales de la salud. El sujeto de la atención 
es la persona, por lo tanto el respeto a su dignidad, el valor de su vida, los derechos que tiene como ser humano a la salud, al bienestar, la seguridad, la paz, la educación, el trabajo, la recreación, etc. son directrices que orientan la dimensión técnico-científica y ética de esta atención.

Cuando se hace referencia a la atención, se entiende ésta como el cuidado que se brinda a la persona, en forma integral, a través de la interacción profesional-persona y no como el cuidado de la enfermedad. Se ofrecen cuidados a la persona para ayudarla a mantenerse saludable, o para ayudarla a afrontar los riesgos o manifestaciones de un problema de salud, o para adaptarse a vivir con limitaciones.

Si la atención en salud se centra en el valor, en la dignidad de la persona, en sus necesidades y derechos, y no en la enfermedad y la tecnología de diagnóstico y tratamiento, ésta tiene su verdadero sentido y la persona se siente atendida, porque se hacen evidentes los elementos de la ética de la atención tales como la interacción, el conocimiento, el deber profesional y la fuerza moral. ${ }^{14}$

La interacción es dialógica, simétrica entre el profesional y la persona atendida. Esta interacción se construye a partir de la confianza y progresa con el respeto y el afecto; en dicha interacción se tienen en cuenta los valores de las personas involucradas. ${ }^{11,14}$

Entonces ante el paciente la actitud que adopte el profesional de la salud, debe estar matizada de tolerancia, sensibilidad, respeto y amor frente a ese ser humano; es bueno insistir en que no solamente son importantes los conocimientos, las habilidades, el dominio de técnicas y destrezas, sino que se requiere además de una actitud recta y una comunicación con la familia y con los demás integrantes del equipo de salud, para lograr satisfacer las necesidades de la persona a quien se atiende. ${ }^{15}$

El saludar y presentarse a la persona enferma debe ser señal clara de reconocimiento como ente individual y único. La cortesía y una sonrisa favorecen el acercamiento, confianza y seguridad para alguien que se enfrenta a una situación nueva, llena de incertidumbre, estrés, angustia y expectativa, como es la enfermedad.

Mirar al interlocutor, establecer un contacto visual con él, es el paso inicial de una efectiva relación interpersonal. El tacto es parte importante del lenguaje, un simple apretón de manos, una palmada en el hombro, pueden calmar y tranquilizar a una persona ansiosa.

Por otra parte la información sencilla, clara y exacta sobre el paciente, el actuar con rapidez, con decisión y manteniendo la calma, especialmente en caso de emergencia, transmite seguridad y confianza tanto al paciente como a su familia.

La prudencia es fundamental en los comentarios que se hacen frente a la persona enferma.

Cada uno responde de manera diferente ante la enfermedad. Es el profesional de la salud, quien debe tratar de conocer y comprender la situación y respuesta de cada uno para poder ofrecer apoyo, reforzar las defensas, hacerlo sentir protegido y seguro, responder a las preguntas que formule, darle la información precisa y comprensible.

Es útil y beneficioso crear es- pacios y animar al paciente para que exteriorice sus sentimientos y reflexiones, permitirle que exprese lo que siente: miedo, ira, culpa, ansiedad, tristeza, con el fin de disminuir la frustración causada por su situación de salud.

Es importante además llamar al paciente por su nombre y no con calificativos estereotipados de presunta "ternura", como abuelita, madrecita, gordita, mi amor, los cuales dichos completamente fuera de su verdadero contexto, son términos que en algunos casos pueden llegar a desorientar y siempre afectan la autoestima, aparte de que podría ser una falta de respeto con el paciente. En cambio cuando se identifica a alguien por su nombre se le hace sentir valorado como persona, se le individualiza, se le hace sentir importante, más seguro y con mucho menos frialdad. ${ }^{11,15}$

La invasión por parte de la tecnología en los diferentes servicios, en donde la vida completa está en manos del personal que brinda cuidado, los conocimientos científicos, los descubrimientos y avances tecnológicos, son importantes, pero es importante hacer énfasis, en que no se puede permitir que la intervención se centre únicamente en el cuidado de sondas, equipos, catéteres, el cuidado debe ir mucho más allá; el cuidado debe estar centrado en el paciente como persona, y a su alrededor está la tecnología, el equipo de salud, la familia, etc.

Se debe velar por el ambiente mediante medidas que contribuyan a la disminución del ruido, la disminución de la luz innecesaria, con las cuales se favorece la disminución de la angustia y el estrés que inevitablemente genera el hecho de estar hospitalizado. 
Igualmente es tranquilizante el amable contacto personal, el tomarlo de la mano, el afecto generado por el contacto físico, las explicaciones con voz firme pero moderada, así parezca que no escucha, también contribuyen a generar tranquilidad.

Se debe ser cuidadoso: la persona enferma no necesita que se le exprese compasión y piedad, sino todo lo contrario, necesita palabras positivas dentro de una actitud igualmente positiva que genere energía y lo estimule en su proceso de recuperación, al tiempo que se le ayuda a ver que el dolor engrandece y fortalece, mostrándoselo de una manera positiva o ayudándole a profundizar en el sentido trascendente que el paciente le ve. ${ }^{16}$

\section{Conclusiones}

Para hablar de una atención humana en los diferentes escenarios del área de la salud, es importante contemplar los agentes que participan en ella, esto es, médicos, enfermeras, terapistas, nutricionistas, entre otros, instituciones prestadoras de servicio y formadoras, y los atributos que deben caracterizar dicha atención.

Respecto a los profesionales su responsabilidad se centra en garantizar la calidad de su desempeño a quienes reciben sus servicios. Esto implica velar por la continuidad y actualización permanente en su formación; así como velar porque se pueda contar con la infraestructura necesaria a nivel de recursos humanos y materiales. A la vez las instituciones responderán por proveerlos, motivar a sus profesionales en su desempeño y en su capacitación competente.

La práctica de los profesionales de la salud en la atención de los pacientes muestra una fuerza moral, que se deriva de los valores y los principios de ética que deben aplicar en su desempeño profesional, dentro de los cuales está el respeto desarrollado en doble vía: profesional-paciente y pacienteprofesional.

El conjunto de profesionales e instituciones asistenciales y formadoras, favorecerá que la atención humana se caracterice por estar matizada de valores éticos y morales dentro de los cuales el respeto ocupa un lugar privilegiado.

\section{Referencias Bibliográficas}

1 Rodriguez A. Hacia la humanización en salud. ARS Médica. 2003; 10(10).

2 Congreso de Colombia. Ley 911, Profesión de Enfermería. Título I. Artículo 1. Diario Oficial. 2004.

3 Ariza C. Caballero R. Calidad del Cuidado de Enfermería al paciente hospitalizado. Bogotá. Colombia:Editorial Javeriana Pontificia Universidad Javeriana. 2008. $99 \mathrm{p}$.

4 López N. Persona humana. Instituto de Ciencias para la familia. España: Universidad de Navarra. 2002.

5 Posada N. Ser Humano y Persona. Bogotá, Colombia: Consejo Episcopal Latinoamericano. Universidad de La Sabana. 2003. 212 p.

6 Melendo T. Aproximación al concepto de "persona". España: Universidad de Málaga. 2001.

7 Melendo T. Las dimensiones de la persona. Madrid, España:Biblioteca Palabra. 2a Edicion. 2001. 174 p.

8 Cerda I. La ciencia y la tecnología en la humanización de los servicios de salud. Rev. Medicina, Vida y Salud. Marzo, 2008; 2(3): p. 3

9 Oldnal A. Paterson \& Zderad's. Humanistic Nursing Theory. JAN
[Internet] 2008, Julio; 18(11): [1850] Disponible en: http://bit.ly/ KGw6M3

10 Kleiman S, Paterson J, Zderad L. Humanistic nursing theory with clinical applications. In: Parker ME. Nursing theories and nursing practice. Philadelphia F.A.: Davis Company, 2005. p. 152-168.

11 Ariza C. Cuidado de Enfermeria al paciente en postoperatorio temprano de una revascularización miocárdica [Tesis Doctoral]. Bogotá. Facultad de Enfermería. Universidad Nacional de Colombia. 2009.

12 Llano C. Dilemas éticos de la empresa contemporánea. México: Fondo de cultura Económica 1997. 313 p.

13 Ariza C. La Excelencia del cuidado. Un reto para Enfermería. Actualizaciones de Enfermería. Departamento de Enfermería. Fundación Santa Fe de Bogotá. 2006.

14 Ariza C. ¿Es posible dar un cuidado humano en la UCI?. 1er Congreso Nacional de Cuidado Crítico. Clínica Universitaria Teletón. Universidad de La Sabana. Febrero. 2010.

15 Garzón N. Ética en el cuidado de enfermería. Dimensiones del Cuidado. Colombia: Facultad de Enfermería. Universidad Nacional de Colombia. 1998.

16 Watson J. Caring science as sacred science. Philadelphia: FA Davis Company. 2005. 


\section{Enfermería Universitaria}

\section{|Lineamientos para Autores}

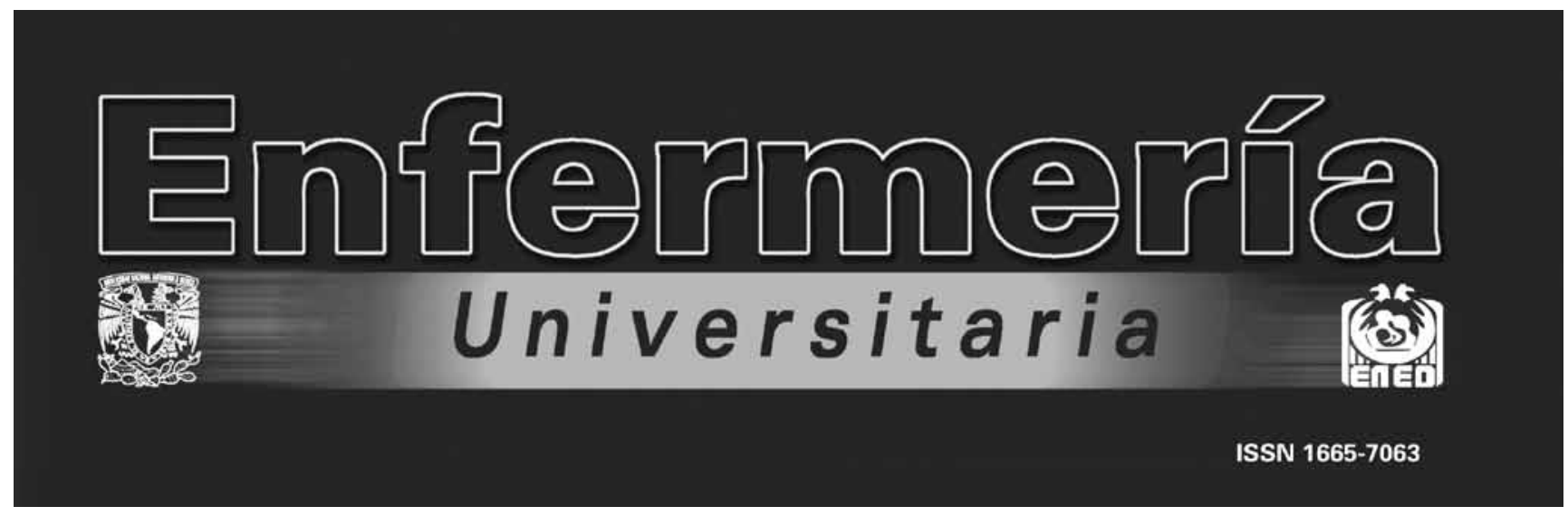

La revista Enfermería Universitaria es una publicación trimestral de la Escuela Nacional de Enfermería y Obstetricia de la UNAM, está indizada en la base de datos CUIDEN de Granada, España, y en el Sistema de Información LATINDEX del Dpto. de Bibliografía Latinoamericana de la Dirección General de Bibliotecas de la UNAM. Tiene como objetivo difundir aportaciones en materia de investigación en salud, educativa y práctica profesional, busca contribuir al fortalecimiento, discusión, e intercambio disciplinar e interdisciplinar con instituciones de salud y académicas, del ámbito nacional e internacional. Pueden participar como autores: profesionales, profesores, y estudiantes de enfermería, y de otras disciplinas de la salud afines.

Políticas de Publicación. La Revista considera para su publicación artículos inéditos, reservándose los derechos exclusivos sobre los mismos. Los conceptos, juicios y opiniones expresados en los artículos serán responsabilidad de las y los autores y no reflejan los criterios, políticas o puntos de vista de la institución y la Revista. La recepción de un trabajo no implica la obligación de publicarlo, ni compromiso con respecto a la fecha de su aparición. Los autores son responsables del contenido de cada uno de los artículos incluyendo cualquier cambio sugerido por el comité revisor.
Proceso de selección. Las contribuciones recibidas pasan por un proceso de selección que dura aproximadamente 4 meses:

1. Dictamen editorial: a la recepción del manuscrito se verifica el cumplimiento de los lineamientos definidos por la revista 
y descritos en las indicaciones a los autores.

2. Dictamen académico: seguidamente se examina el valor académico y científico del documento mediante un sistema de arbitraje por pares (peer review) realizado por expertos en las distintas materias, nacionales y/o internacionales, externos al Comité Editorial. El editor designa dos árbitros y en caso que sus dictámenes discrepen uno del otro se asigna un tercero. Siendo los dictámenes: "Aprobado", "Aprobado con modificaciones" (Se da 1 mes desde su notificación al autor para reenvío con un máximo de 3 reenvíos ) y "No aprobado". En esta fase se protege el anonimato tanto de autores como de revisores.

3. Comité Editorial: con base en los lineamientos de la revista, el valor académico o científico del artículo y la opinión de los árbitros, se define si se publica el artículo o documento presentado.

Las colaboraciones que se reciben deben ser originales e inéditas y responder a alguna de las siguientes secciones:

Editorial. En esta sección un experto opina, analiza y reflexiona sobre un tema novedoso que refleje su experiencia, o bien puede estar dirigido a comentar artículos publicados o a los temas tratados en el número respectivo de la revista.

Artículos de Investigación. Son documentos que tienen como objetivo difundir resultados de investigación original e inédita. Pueden ser investigaciones cuantitativas (se estudia la asociación o relación entre variables cuantificadas) o cualitativas (se centra en el entendimiento de contextos estructurales y situacionales). Pueden ser en formato breve según la concreción de objetivos y resultados.

Artículos de revisión. Tienen como objetivo examinar la investigación publicada sobre un tema en un periodo delimitado y ubicarla en una perspectiva determinada.

Innovación para la práctica. Dentro de esta sección pueden ser incluidos artículos que den a conocer experiencias, reflexiones o propuestas originales, innovadoras y/o relevantes dentro del campo del cuidado, de la gestión (o administración), de la propia investigación o en la educación en enfermería, que hayan marcado o contribuido de manera clara y/o determinante en cambios en esta disciplina.

Cartas al editor. Son cartas sobre temas relevantes para la enfermería o bien relacionadas a los artículos publicados en la revista. Los autores de artículos objeto del comentario, tendrán derecho de réplica.

\section{Preparación del manuscrito}

Entregar impresión original y dos copias en papel bond tamaño carta, letra tipo arial 12, a doble espacio, con paginación inferior lateral derecha empezando con la carátula. Cada parte del escrito en hojas separadas: carátula, resúmenes y palabras clave, texto del artículo, referencias, cuadros, gráficas. Además grabación en $\mathrm{CD}$, con nombres de los archivos y especificación del formato.
El trabajo se acompañará de una carta del autor principal especificando que los manuscritos son inéditos y que no se han enviado simultáneamente a algún otro medio, ni se enviarán antes de conocer la decisión de la Revista. Los autores adjuntarán una declaración firmada indicando que si el documento se acepta para publicación, los derechos de reproducción son propiedad exclusiva de la revista Enfermería Universitaria.

\section{- Carátula}

- Título completo en español y en inglés con extensión máxima de quince palabras que refleje en forma específica y clara el contenido, sin abreviaturas, siglas y sin signos de admiración, interrogación o comillas.

- Título breve en español con extensión máxima de ocho palabras.

- El número máximo de autores para artículos de investigación es seis, y en formato breve tres. El nombre y los apellidos de cada autor se registrarán inmediatamente debajo del título; en notas separadas al pie de la página se anotarán los títulos académicos y cargos de los autores, así como el nombre de la institución de adscripción, la dirección, teléfono, fax y dirección electrónica del autor responsable de la correspondencia y agradecimientos, si es el caso. - Incluir información acerca de cualquier beca o financiamiento recibido.

\section{- Resumen y palabras clave}

Cada manuscrito se acompañará de un resumen en español y en inglés con una extensión no mayor a 250 


\section{Enfermería Universitaria}

palabras, para artículos de investigación señalar: objetivo, metodología, resultados, discusión y conclusiones. Si se trata de otros artículos, el resumen debe incluir: el propósito u objetivos, síntesis del contenido y conclusiones y que no exceda de 200 palabras.

El resumen debe estar seguido por tres a diez palabras clave o descriptores en español (DeCS) y en inglés (MeSH), que facilitan la indexación de los artículos. Para determinarlos se pueden consultar las páginas http://decs.bvs.br/ o http: www.nlm.nih.gov/mesh/MBrowser.html

\section{- Cuerpo del trabajo según sección.}

1. Editorial. Su extensión puede ser entre 2 y 5 cuartillas.

2. Artículos de investigación. Extensión total de 20 cuartillas; para manuscritos en formato breve un máximo de 10 cuartillas. Tanto los trabajos de tipo cuantitativo o cualitativo incluirán los mismos apartados:

- Introducción: Incluir el objetivo y justificación del trabajo con fundamentos teórico-conceptuales. En estudios cualitativos precisar la perspectiva teórica seleccionada.

\section{Metodología:}

Cuantitativos.- En esta sección incluir la descripción de la población de estudio, tamaño de muestra, tipo de muestreo, criterios de selección y pérdida de sujetos. Describir en forma clara y precisa la operacionalización de variables, medición, plan de análisis y métodos estadísticos utilizados, permitiendo así la verificación de los resul- tados. Presentar las pruebas de validez y confiabilidad, de los instrumentos de medición, definir los términos estadísticos, abreviaturas y los símbolos e indicar los programas informáticos utilizados. Cuando se trata de estudios con seres humanos, precisar si los procedimientos empleados han respetado los criterios éticos (Declaración de Helsinki). Adjuntar, en su caso, carta de consentimiento informado y aprobación del comité de ética institucional

Cualitativos.- Brindar información sobre el lugar y contexto del estudio, la selección de los participantes, cómo fueron recolectados los datos, y el tipo de investigación según la posición del investigador, así como los aspectos éticos de la investigación. Descripción del proceso de análisis y de selección de testimonios y de datos contradictorios, sesgos, y participación en el análisis de equipo y participantes.

\section{Resultados:}

Cuantitativos.- Presentarlos con secuencia lógica en el texto. Enfatizar o resumir sólo los hallazgos relevantes y no repetir en el texto los datos de los cuadros, figuras e ilustraciones. Cuidar que el orden y secuencia de éstos sea el correspondiente a la presentación de los mismos.

Cualitativos.- Presentar en forma explícita los resultados, en forma tal que se brinde una imagen significativa del fenómeno bajo estudio.

- Discusión y conclusiones: Se derivan del análisis de los resultados y propósitos del estudio, destacar los aspectos nuevos o relevantes, así como la explicación a los hallazgos encontrados. Comparar los resultados con otros estudios similares, vincular las conclusiones con los objetivos de estudio y cómo contribuye al campo del conocimiento de enfermería. Las afirmaciones deben estar plenamente respaldadas por los datos mostrados, además mencionar las limitaciones del estudio. Para estudios cualitativos es importante que se establezcan las conexiones teóricas pertinentes.

3. Artículos de revisión. Debe participar al menos una persona con experiencia previa en el tema, hacer explícito el universo que cubre esta revisión en cuanto a periodo y bases de datos consultadas, incluye: introducción (con objetivo explícito), desarrollo y conclusiones, en éstas queda explícita la contribución de la revisión en aspectos de la práctica o nuevos elementos de investigación, deben contener mínimo 40 fuentes bibliográficas, pueden incluirse cuadros y figuras,

4. Innovación para la práctica en las siguientes modalidades:

- Información relevante para la práctica. Brindar el contexto de la información, es decir de dónde surge y a quién va dirigida. Información a la que alude (normas, procedimientos, acuerdos, lineamientos, guías clínicas etc.) y fuente(s) de la información.

- Experiencias de la práctica. Introducción, estrategia(s), experiencias, intervención(es), plan de cuidados (si fuera el caso), resultados, conclusiones, referen- 
cias; al menos 5 artículos publicados y 5 textos relativos al tema.

- Revisión crítica de un estudio publicado. Incluirá: Título del comentario, pregunta breve que deberá quedar resuelta al final del resumen, referencia completa del artículo que se comenta, el abstract o resumen del artículo tomado como referencia, comentario realizado por un revisor experto en la materia, explica los motivos por los que se seleccionó el artículo o la importancia de los hallazgos para un área concreta. Citas bibliográficas en las que el revisor base sus comentarios.

- Ensayos. Introducción, desarrollo, conclusiones, referencias; las necesarias para apoyar el manuscrito, preferentemente publicadas en los últimos cinco años.

5. Cartas al editor. Su extensión tendrá un máximo de dos cuartillas.

\section{Cuadros y Figuras.}

$\mathrm{Al}$ diseñar cuadros (tablas) y figuras (dibujos, gráficos y fotografías) tener en cuenta una posible reducción de tamaño. También es importante mencionar que las imágenes o fotografías adquiridas vía internet, no mantienen la resolución requerida para procesarse por lo que debe evitarse su uso. Los encabezados de cuadros y las leyendas de figura deberán entenderse sin hacer referencia al texto. Los pies de figura están señalados con los números arábigos conforme a la secuencia global que les corresponde. El material gráfico constituido por tablas, cuadros y figuras deben presentarse en el orden en que se citan en el trabajo (Tabla 2; Figura 4), en hojas separadas y en el caso de utilizar gráficas estas deberán contar con alta resolución preferentemente archivos JPG.

\section{Referencias Bibliográficas}

Deben seguir estrictamente el modelo Vancouver ${ }^{1}$ utilizado por la mayoría de las revistas biomédicas. Las referencias bibliográficas se numerarán consecutivamente en el orden en que aparecen en el texto y cada una corresponderá a la fuente citada en la lista de referencias bibliográficas al final de la publicación. El número que se le asigna a cada referencia bibliográfica en el texto debe colocarse en números arábigos en superíndice. Una vez que la fuente ha sido citada, el mismo número se utiliza en todas las referencias posteriores. La asignación de los números deben convertirse en identificadores únicos de esa fuente y se reutilizan cada vez que la referencia se cite a lo largo del texto. Para mayor información se recomienda consultar los ejemplos disponibles en Fisterra. com: Estilo Vancouver: Requisitos de uniformidad para manuscritos enviados a revistas biomédicas. http://bit.ly/HTQQcW. A continuación se proporcionan algunos ejemplos para diferentes tipos de documentos.

Libros. Autor/es. Título. Número de Edición. Ciudad de publicación: Editorial; año de publicación. Ejemplo: Burke MM, Walsh MB. Gerontológica: cuidados integrales del adulto mayor. $2^{\text {a }}$ ed. Madrid: Harcourt Brace; 2000.

Capítulo de libro. Autor/es del capítulo. Título del capítulo. En: Editores ó Coordinadores del libro, Título del libro. Número de edi- ción, Ciudad de publicación: Editorial; año de publicación. Páginas inicial y final correspondientes al capítulo. Ejemplo: Jack MQ. Cuidados intensivos en pacientes neurológicos. En: Marino ANET, editores. Cuidados Intensivos Médicos. $3^{a}$ ed México: Interamericana Mc Graw-Hill; 1998. p. 351-366.

Artículos de Revistas. Autor/es. Título del artículo. Abreviatura internacional del título de la revista Año; volumen (número): página inicialfinal del artículo. Ejemplo: Barraza K, Villareal A, Seipp C, Lara P, et al. Prevalencia de asma y otras enfermedades alérgicas en niños escolares. Salud Publica Mex 2005; 43 (3): 433-477.

Algunos autores acostumbran incluir sus dos apellidos, en ese caso citarlos enlazados con un guión ejemplo: Barraza-Colin M, Melgarejo-Ortíz A.

Para las abreviaturas internacionales de los títulos de las revistas se recomienda consultar en: http://1. usa.gov/mHKV2x http://bit.ly/HWbYnP

Documentos Electrónicos. Los documentos tomados de internet citados y referenciados deben cumplir con los siguientes criterios de calidad:

Estar alojados en sitios Web fiables que garanticen estabilidad del contenido con el paso del tiempo; que presenten actualización del sitio; que sean de fácil navegación; que presenten enlaces a otras páginas del sitio y a otros sitios Web con información relacionada y que proporcionen datos de contacto como e-mail, dirección postal, entre otros. 


\section{Enfermería Universitaria}

El documento debe traer fecha de publicación y actualización.

Las citas y las referencias deben presentar consistencia.

El documento debe ser a texto completo y terminado.

La indicación del tipo de documento electrónico se registra entre corchetes. Ejemplo: [Internet]

Para los documentos en línea la fecha de consulta se registra antecedida de la palabra acceso y entre corchetes. Ejemplo: [Acceso 18 de noviembre de 2001]

\section{Artículo de revista de Internet}

Autor/es del artículo. Título del artículo. Título abreviado de la revista [Internet].año mes [fecha de consulta]; volumen (número): [páginas si aparecen].Dirección electrónica. Ejemplo:

Alconero AR, Casaus M, Iglesias R, De la Friera V, Noriega MJ, Fadón A. El corazón de los jóvenes. Enferm Cardiol [Internet]. 2006 [acceso 23 de marzo de 2008]; 13 (38): [13-18]. Disponible en: http:// bit.ly/NYsbYe

\section{Monografía en Internet}

Autor/es o Director/ Coordinador/ Editor. Título [Internet].Edición. Lugar de publicación: Editorial; año [fecha de consulta].Dirección electrónica

\section{Referencia bibliográfica.}

1. Rodríguez- Bonache MJ. ¿Cómo se debe citar en un artículo científico? Rehabilitación (Madr) 2002; 36(2):67-69.

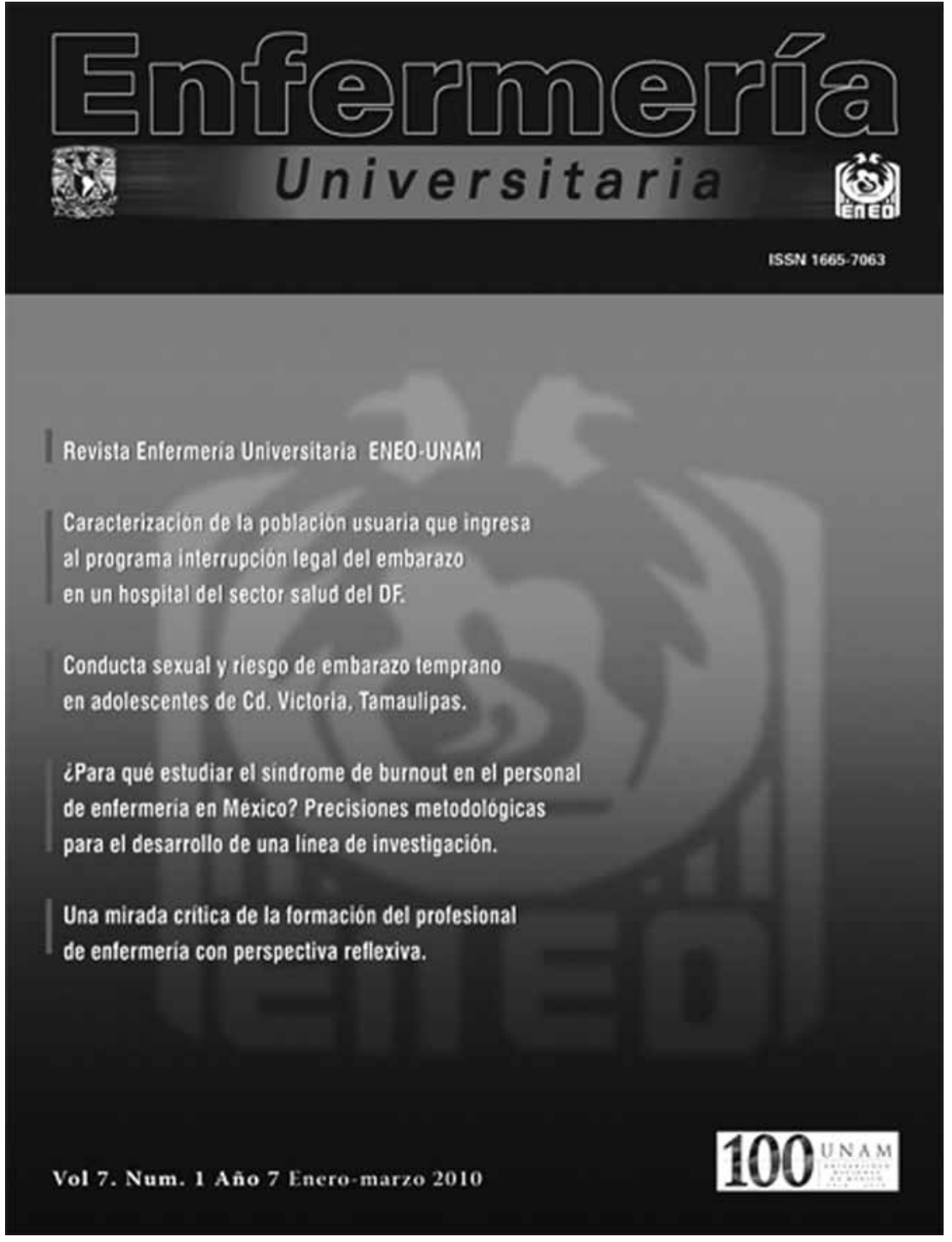

Caracterizacion de la poblacion usuaria que ingresa

al programa interrupcion legal del embarazo

Conducta sexual y riesgo de embarazo temprano

en adolescentes de Cd. Victoria, Tamaulipas.

¿Para que estudiar el sindrome de burnout en el personal

de enfermeria en México? Precisiones metodologicas

para el desarrollo de una linea de investigación.

Una mirada critica de la formación del protesional

de enfermeria con perspectiva reflexiva.

Vol 7. Num. 1 Año 7 Enero-marzo 2010 